\title{
A Physiologically-Based Adaptive Three-Gaussian Function Model for Image Enhancement
}

\author{
Zilong Xu, Kaifu Yang, Yongjie $\mathrm{Li}^{*}$ \\ Key Laboratory for Neuroinformation of Ministry of Education, School of Life Science and Technology, \\ University of Electronic Science and Technology of China, Chengdu, China \\ Email: ${ }^{*}$ liyj@uestc.edu.cn
}

Received 1 December 2014; accepted 25 December 2014; published 13 January 2015

Academic Editor: Prof. Zhongzhi Shi, Institute of Computing Technology, Chinese Academy of Sciences, China

Copyright @ 2015 by authors and Scientific Research Publishing Inc.

This work is licensed under the Creative Commons Attribution International License (CC BY). http://creativecommons.org/licenses/by/4.0/

cc) (i) Open Access

\begin{abstract}
Image enhancement is an important pre-processing step for various image processing applications. In this paper, we proposed a physiologically-based adaptive three-Gaussian model for image enhancement. Comparing to the standard three-Gaussian model inspired by the spatial structure of the receptive field (RF) of the retinal ganglion cells, the proposed model can dynamically adjust its parameters according to the local image luminance and contrast based on the physiological findings. Experimental results on several images show that the proposed adaptive three-Gaussian model achieves better performance than the classical method of histogram equalization and the standard three-Gaussian model.
\end{abstract}

\section{Keywords}

Image Enhancement, Receptive Field, Visual System, Three-Gaussian Model

\section{Introduction}

Images play an important role in transferring information. In order to obtain more information from collected images, image enhancement techniques are commonly required to improve image quality. Traditional image enhancement methods can be roughly divided into two categories: 1) spatial domain methods, such as gray-level transformation, piecewise-linear transformation, and histogram equalization, etc. 2) frequency domain methods,

*Corresponding author.

How to cite this paper: Xu, Z.L., Yang, K.F. and Li, Y.J. (2015) A Physiologically-Based Adaptive Three-Gaussian Function Model for Image Enhancement. International Journal of Intelligence Science, 5, 72-79.

http://dx.doi.org/10.4236/ijis.2015.52007 
which include high-pass filtering, high-frequency emphasizing filtering, and homomorphic filtering etc. However, these methods mentioned above are in general difficult to balance well among various requirements of image quality, such as contour enhancement, dynamic range, denoising and so on. In addition, the ability of traditional image enhancement methods is far behind the human visual system in almost all aspects.

Early physiological studies have revealed that the retinal ganglion cells have a receptive field (RF) consisting of concentric regions, i.e., an approximately circular center and an annular surround [1] [2]. DOG model was proposed by Rodieck to describe the classical receptive field (CRF) of the ganglion cells [1]. Li et al. found that the cells at some distance from the contrast borders were less affected, while the border enhancement might become quite stronger when the centre was close to the corner of a bright contour [2]. Ramachandran found that the luminance gradients of an area are essential for producing perception of three-dimensional visual scenes [3]. By analyzing the length-response functions of lateral geniculate neurons in the cat, Li et al. have demonstrated an extensive disinhibitory region (DIR, i.e., non-CRF) outside the classical inhibitory surround of the receptive field [4]. According to this finding, a three-Gaussian function model was proposed in [5]. By setting appreciate parameters of the three-Gaussian model, good fit could be obtained almost for all data that show disinhibitory phenomenon [5]. Functionally, the three-Gaussian model can not only enhance the edge information but also transmit brightness information with low spatial frequency [5] [6].

However, the three-Gaussian model cannot dynamically adjust its parameters according to the local stimulus. In fact, the adaptation to the stimulus features (e.g., the luminance contrast) of the receptive field in the visual system has been deeply studied [7]-[12]. Some experiments [7] showed that the responses of retinal ganglion cells first increased abruptly, and then decayed exponentially to a lower value following the abrupt increase in stimulus contrast. Based on extracellular recordings from 69 LGN cells in the anesthetized cat, Nolt et al. found that the spatial summation within their receptive fields was dependent on the contrast of the stimuli presented. They reported that the contrast-dependency in the retinal ganglion cells directly resulted from a reduction in the size of the center mechanism due to an increase in contrast [8]. By characterizing the adaptation of neurons in the cat lateral geniculate nucleus (LGN) to changes in stimulus contrast and correlations, Lesica et al. found that the space constant of the excitatory center increased with a decreasing in stimulus contrast [9]. In addition, it has been shown that spatial summation in the primary visual cortex of the cat and monkey is strongly dependent on stimulus contrast; the area (length and width) over which responses summate generally increases as the stimulus contrast decreases; fitting summation curves with a DOG model shows that this contrast-dependent spatial summation seems to derive from a change in the actual size of the receptive field [10]-[12].

To simulate the dynamic properties of the RF, in this work we present an adaptive three-Gaussian function model to automatically adjust its parameters according to the properties of local stimulus, i.e., local contrast and luminance, for image enhancement.

\section{Computational Model}

\subsection{Three-Gaussian Function Model}

Figure 1 shows the model of three-Gaussian model, in which the center and surround denote respectively the excitatory region (Center) and inhibitory region (Surround) of CRF. The disinhibitory region usually covers a larger range of visual field. The response amplitude of cells with stimulus radius is shown in the top-right corner of Figure 1. The three-Gaussian function model is described as [5]

$$
f(x, y)=A_{1} \mathrm{e}^{\frac{-\left(x^{2}+y^{2}\right)}{\sigma_{1}^{2}}}-A_{2} \mathrm{e}^{\frac{-\left(x^{2}+y^{2}\right)}{\sigma_{2}^{2}}}+A_{3} \mathrm{e}^{\frac{-\left(x^{2}+y^{2}\right)}{\sigma_{3}^{2}}}
$$

where $A_{1}$ and $\sigma_{1}$ are the strength and space constant of the excitatory center, $A_{2}$ and $\sigma_{2}$ are the strength and space constant of the inhibitory surround, and $A_{3}$ and $\sigma_{3}$ are the strength and space constant of the disinhibitory outer-surround region.

This three-Gaussian model assumes that the sensitivity profiles of the three regions (i.e., center, surround and outer-surround) are distributed as Gaussians, which are circularly concentric with their peaks overlapped at the center point of the RF center region. This model also assumes that the three parts summate linearly from all parts of the receptive field. Functionally, the combination of the first two Gaussians (i.e., the DOG model) serves to detect and enhance the edges, and the third Gaussian serves for the brightness information transmission by compensating the loss of the low frequency components resulted from the almost balanced center and sur- 
round mechanisms in the DOG model of most cells [5].

\subsection{Adaptive Mechanism}

Based on the experimental findings about the stimulus-dependant RF properties, we specifically introduce two dynamic RF features: 1 ) the excitatory strength of the center $\left(A_{1}\right)$ increases nonlinearly with the increasing of the local contrast; 2$)$ the inhibitory space constant $\left(\sigma_{2}\right)$ of the RF surround is decreased with the increasing of the local luminance.

In this paper, we define the feature of local luminance contrast as the standard deviation within a small image patch around each pixel in the image. We denote local luminance contrast as Con . In addition, we use a modified sigmoid function to simulate the nonlinear transformation of neural information. The relationship between $A_{1}$ and $C o n$ is experimentally defined as

$$
A_{1}=6+\frac{1}{1+\mathrm{e}^{-10 \times(\text { Con }-0.5)}}
$$

Figure 2 shows the relationship of center excitation $\left(A_{1}\right)$ along with the local contrast ( Con ).

On the other hand, in order to improve the contrast of shading regions of the image, another parameter $\left(\sigma_{2}\right)$ is adjusted with local luminance. In our adaptive three-Gaussian function model, we can reduce inhibitory space constant $\left(\sigma_{2}\right)$ to weaken the surround inhibition when the luminance value of the pixel to be processed is high.

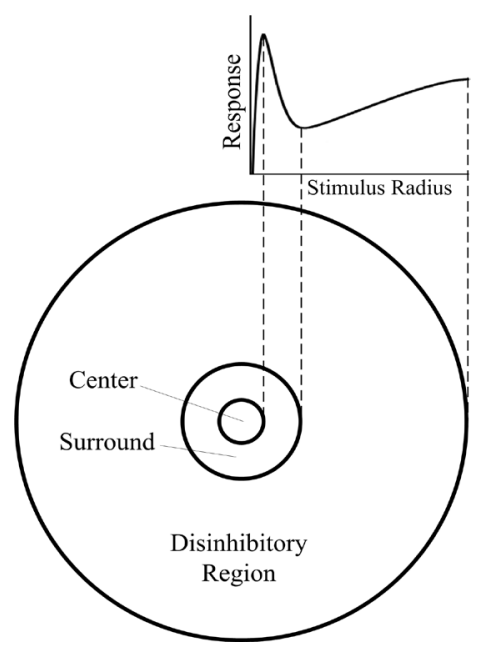

Figure 1. The spatial strucutre of the receptive field of the ganglion cells and LGN cells.

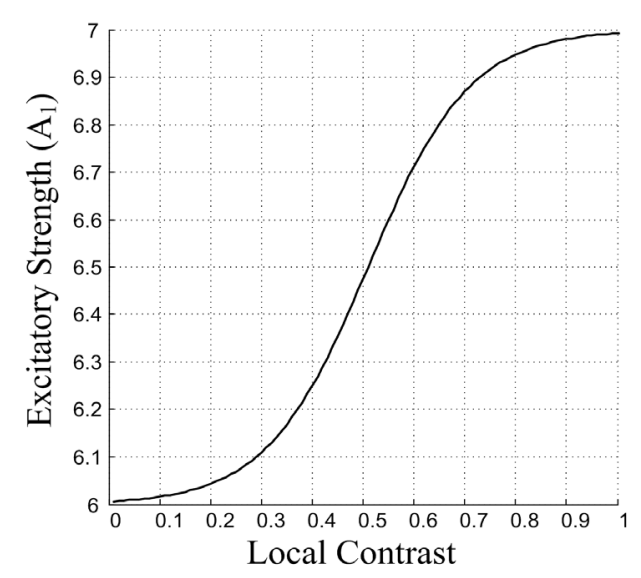

Figure 2. The relatinship of center excitatory $\left(A_{1}\right)$ along with local contrast (Con). 
We use $L$ to denote local luminance value of each pixel in the image. Similar to Equation (2), we also employ a modified sigmoid function to represent the relationship between the inhibitory space constant $\left(\sigma_{2}\right)$ and the local luminance feature. The relationship between $\sigma_{2}$ and $L$ is experimentally defined as

$$
\sigma_{2}=1.25+\frac{0.1}{1+\mathrm{e}^{10 \times(L-0.45)}}
$$

Figure 3 shows the relationship between $\sigma_{2}$ and $L$. Note that a simple smoothing filtering is applied on the map of $C o n$ and $L$ to removing noises.

As described in Equations (2) and (3), the kernel idea of our adaptive three-Gaussian model is that two important parameters (i.e., $A_{1}$ and $\sigma_{2}$ in Equation (1)) are adaptively adjusted based on the features of local contrast and local luminance, respectively.

It should be pointed out that the curves in Figure 2 and Figure 3 are sigmoid shaped, because sufficient experimental evidence indicates that the change of receptive field properties (e.g., the sensitivity and spatial size) with the stimulus features (e.g., the luminance contrast) seems nonlinear [7]-[12]. Note that the constants in Equations (2) and (3) determining the shapes of the sigmoid curves (e.g., the slope) were experimentally obtained and we have found that these settings are suitable for most of the real-world images, as indicated by several examples shown in the next section.

\section{Results}

In this experiment, we compared our adaptive three-Gaussian method with the popular method of histogram equalization and the standard (non-adaptive) three-Gaussian model. Experimental results on several images are shown in Figure 4, Figure 5, Figure 6, and Figure 7. Note that the zoomed in view of each test image is also listed in Figures 4-7, respectively. From the figures, the results of the standard three-Gaussian model usually include more details than original images, but some regions are over-enhanced (especially in the high contrast place); in addition, the contrast of high-light and shading regions are not enhanced enough. Histogram equalization is efficient in adjusting global dynamic range of images, but it is difficult to obtain good local contrast. In addition, three-Gaussian model usually obtains better performance than histogram equalization.

Our adaptive three-Gaussian function model performs better in both enhancing the local contrast and adjusting global dynamic range. Meanwhile, the proposed method is capable of overcoming the phenomenon of overenhancement. In addition, the performance of our new approach in edge enhancement is much better than the other two methods mentioned above, which can be clearly seen from Figures 4-7, especially from the zoomed in view of each test image.

For quantitative comparison, we employed EME (a measure of enhancement) [13] and SNR (Signal to Noise Ratio) [14] for performance evaluation of image enhancement. SNR is usually defined as the mean target signal to the standard deviation of the noise [13]. In this paper, we define $E$ as the mean value of the all pixels in the

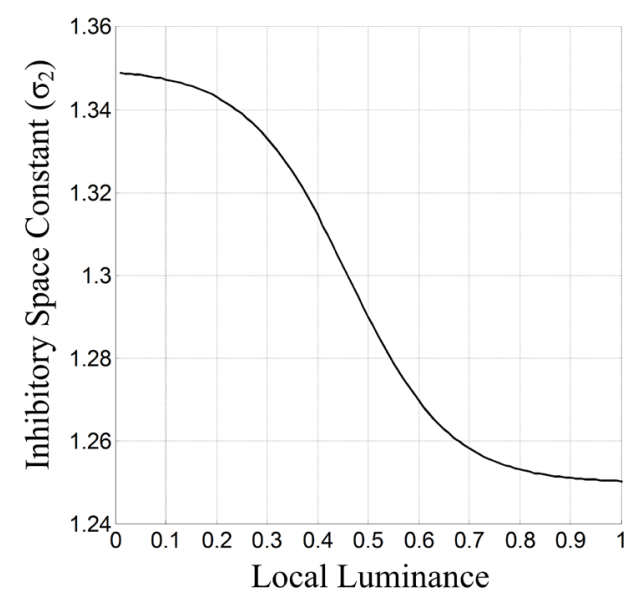

Figure 3. The relatinship of surround space constant $\left(\sigma_{2}\right)$ along with local lumiance $(L)$. 


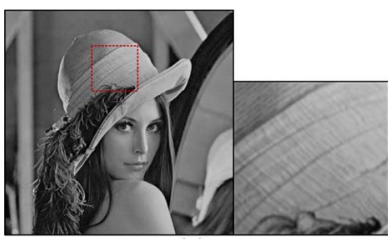

(a)

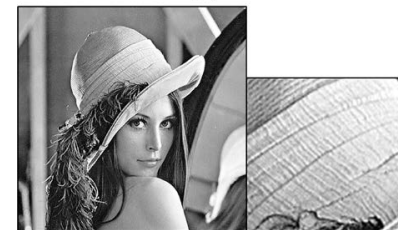

(c)

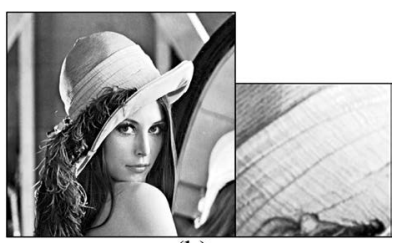

(b)

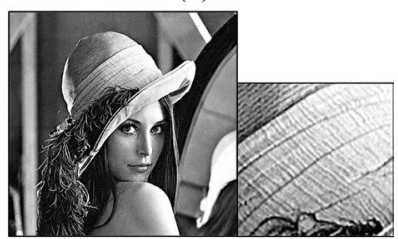

(d)

Figure 4. Results on the Lenna image. (a) Original image; (b) Results of histogram equalization; (c) Results of three-Gaussian function model; (d) Results of the proposed method (adaptive three-Gaussian function model). The zoomed in view of the patch in the red rectangle is also shown for each image.

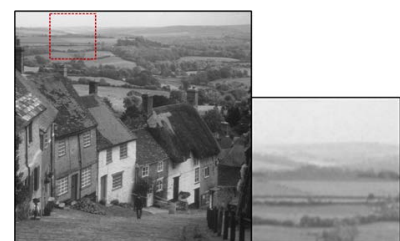

(a)

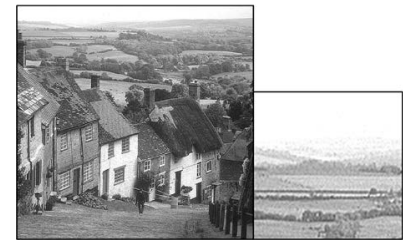

(c)

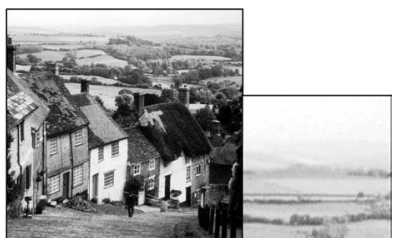

(b)

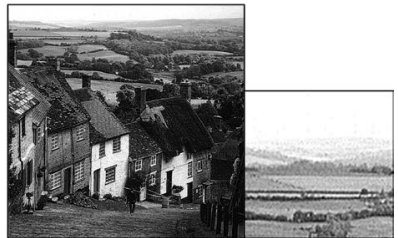

(d)

Figure 5. Results on the Goldhill image. (a) Original image; (b) Results of histogram equalization; (c) Results of threeGaussian function model; (d) Results of the proposed method (adaptive three-Gaussian function model).

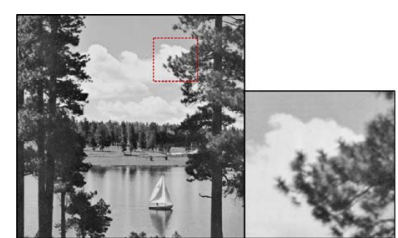

(a)

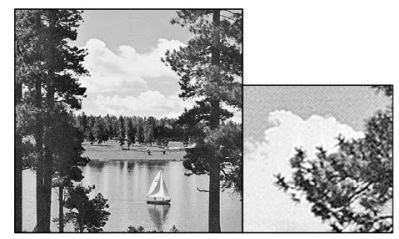

(c)

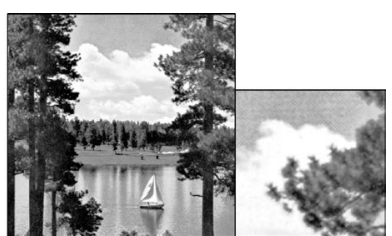

(b)

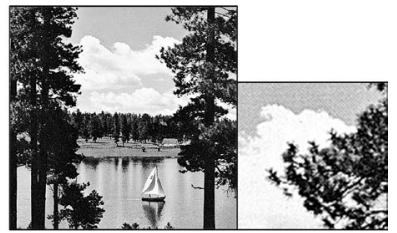

(d)

Figure 6. Results on the Sailboat image. (a) Original image; (b) Results of histogram equalization; (c) Results of threeGaussian function model; (d) Results of the proposed method (adaptive three-Gaussian function model). 


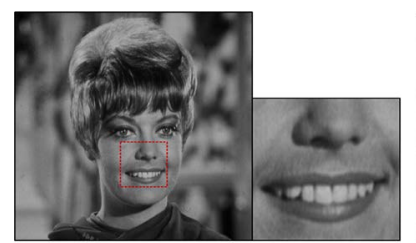

(a)

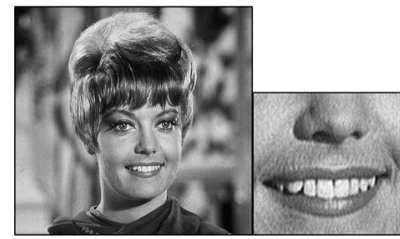

(c)

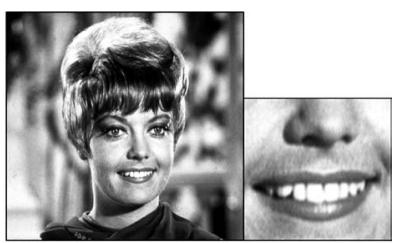

(b)

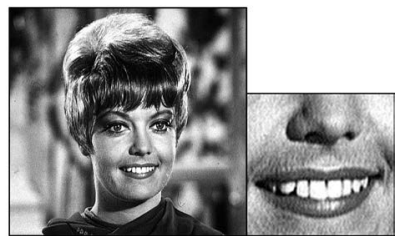

(d)

Figure 7. Results on the Zelda image. (a) Original image; (b) Results of histogram equalization; (c) Results of three-Gaussian function model; (d) Results of the proposed method (adaptive three-Gaussian function model).

image and $\sigma$ as the standard deviation of the all pixels in the image. Therefore, SNR is computed as

$$
S N R=10 \log _{10} \frac{E}{\sigma^{2}}
$$

EME is computed as [14]

$$
E M E=\frac{1}{k_{1} k_{2}} \sum_{l=1}^{k_{2}} \sum_{k=1}^{k_{1}} 20 \ln \frac{V_{\max , k, l}-V_{\min , k, l}}{V_{\max , k, l}+V_{\min , k, l}+C}
$$

where $V_{\min , k, l}$ and $V_{\max , k, l}$ are respectively the minimum and maximum inside a certain block $w(k, l)$ when the whole image is split into $k_{1} k_{2}$ blocks $w(k, l)$ of equal sizes. $c$ is a small constant that equals to 0.0001 to avoid dividing by zero. In general, a higher EME indicates a better enhancement in image details.

EME and SNR of four considered images shown in Figures 4-7 are listed in Table 1 and Table 2. Note that the EME and SNR were calculated from the whole images. From Table 1, the evaluation of EME shows that our adaptive three-Gaussian function model obtains the best performance on edge enhancement. From Table 2, we can see that our new approach achieves competitive performance compared with the standard three-Gaussian function model and histogram equalization in suppressing image noise. This indicates that our adaptive model can well balance the requirements of enhancing edges and inhibiting image noises.

\section{Discussion}

It is generally accepted that the computational image processing methods are far behind the human visual system. They met difficulties to balance well among various requirements of image quality, e.g., contour enhancement and denoising which often cannot be well achieved at the same time. By seeking inspiration from the physiological findings, this paper proposes a physiologically based adaptive three-Gaussian model, which dynamically adjusts the parameters of the three-Gaussian model. The results on several real-world images show that the performance of our new model is better than the standard three-Gaussian function model, especially in overcoming over-enhancement and raising the contrast of highlight and shading regions. Our approach can keep the SNR of an image in an acceptable level; meanwhile, it can effectively enhance the edge profiles and local details of the image. Specifically, in the regions of low luminance, we increase the excitatory strength $\left(A_{1}\right)$ in the regions with high local contrast, which helps enhance the edges with high contrast. Differently, we increase the inhibitory space constant $\left(\sigma_{2}\right)$ in the regions of low brightness, which helps improve the contrast of shading regions.

Our physiologically-based adaptive three-Gaussian function model only simulates the change of inhibitory space constant $\left(\sigma_{2}\right)$ and excitatory strength $\left(A_{1}\right)$ based on the local contrast and local brightness, and don't involve inhibitory strength $\left(A_{2}\right)$ and excitatory space constant, $\left(\sigma_{1}\right)$ which should be improved in the future work. In addition, how to effectively suppress image noise is also an important future direction for us. 
Table 1. EME for the testing images.

\begin{tabular}{ccccc}
\hline \multirow{2}{*}{$\begin{array}{l}\text { Test } \\
\text { Image }\end{array}$} & \multicolumn{3}{c}{ EME } \\
\cline { 2 - 5 } & Original image & Histogram equalization & Three-Gaussian & Adaptive three-Gaussian \\
\hline Lenna & 22.9578 & 55.8410 & 74.2137 & 91.6041 \\
Goldhill & 16.2290 & 40.3469 & 67.6001 & 130.0269 \\
Sailboat & 23.2728 & 40.4264 & 81.2151 & 135.1900 \\
Zelda & 17.1172 & 55.7354 & 67.2700 & 94.1577 \\
\hline
\end{tabular}

Table 2. SNR for the testing images.

\begin{tabular}{ccccc}
\multirow{2}{*}{$\begin{array}{l}\text { Index } \\
\text { Test }\end{array}$} & \multicolumn{3}{c}{ SNR } \\
\cline { 2 - 5 } & Original image & Histogram equalization & Three-Gaussian & Adaptive three-Gaussian \\
\hline Lenna & 64.6106 & 62.6490 & 61.8363 & 61.9148 \\
Goldhill & 64.9061 & 62.4141 & 61.8287 & 61.5272 \\
Sailboat & 62.8929 & 60.6578 & 61.8346 & 60.4178 \\
Zelda & 65.6947 & 61.8349 & 63.3216 & 62.8684 \\
\hline
\end{tabular}

\section{Acknowledgements}

The authors would like to thank Professor Chaoyi Li for his valuable suggestions. The authors also thank the anonymous reviewers for their helpful comments. This work was supported by the 973 project (\#2013CB329401) and the NSFC of China (\#61375115 and \#91120013).

\section{References}

[1] Rodieck, R.W. (1965) Quantitative Analysis of Cat Retinal Ganglion Cell Response to Visual Stimuli. Vision Research, 5, 538-601. http://dx.doi.org/10.1016/0042-6989(65)90033-7

[2] Li, C.I., Chang, Y.J., Chen, P.S., Hsu, H.C. and Wang, H. (1979) Role of Sustained Neurones of Cat Lateral Geniculate Nucleus in Processing Luminance Information. Scientia Sinica, 22, 359-371.

[3] Ramachandran, V.S. (1988) Perceiving Shape from Shading. Scientific American, 259, 76-83. http://dx.doi.org/10.1038/scientificamerican0888-76

[4] Li, C.-Y., Zhou, Y.-X., Pei, X., Qiu, F.-T., Tang, C.-Q. and Xu, X.-Z. (1992) Extensive Disinhibitory Region beyond the Classical Receptive Field of Cat Retinal Ganglion Cells. Vision Research, 32, 219-228. http://dx.doi.org/10.1016/0042-6989(92)90131-2

[5] Li, C.-Y., Pei, X., Zhow, Y.-X. and Von Mitzlaff, H.-C. (1991) Role of the Extensive Area outside the X-Cell Receptive Field in Brightness Information Transmission. Vision Research, 31, 1529-1540. http://dx.doi.org/10.1016/0042-6989(91)90130-W

[6] Keil, M.S., Cristobal, G. and Neumann, H. (2001) A Neurodynamical Retinal Network Based on Reaction-Diffusion Systems. Proceedings of 11th International Conference on Image Analysis and Processing, Palermo, 26-28 September 2011, 209-214.

[7] Smirnakis, S.M., Berry, M.J., Warland, D.K., Bialek, W. and Meister, M. (1997) Adaptation of Retinal Processing to Image Contrast and Spatial Scale. Nature, 386, 69-73. http://dx.doi.org/10.1038/386069a0

[8] Nolt, M.J., Kumbhani, R.D. and Palmer, L.A. (2004) Contrast-Dependent Spatial Summation in the Lateral Geniculate Nucleus and Retina of the Cat. Journal of Neurophysiology, 92, 1708-1717. http://dx.doi.org/10.1152/jn.00176.2004

[9] Lesica, N.A., Jin, J.H., Weng, C., Yeh, C.-I, Butts, D.A., Stanley, G.B., et al. (2007) Adaptation to Stimulus Contrast and Correlations during Natural Visual Stimulation. Neuron, 55, 479-491. http://dx.doi.org/10.1016/j.neuron.2007.07.013

[10] Kapadia, M.K., Westheimer, G. and Gilbert, C.D. (1999) Dynamics of Spatial Summation in Primary Visual Cortex of Alert Monkeys. Proceedings of the National Academy of Sciences of the United States of America, 96, 12073-12078. http://dx.doi.org/10.1073/pnas.96.21.12073 
[11] Song, X.-M. and Li, C.-Y. (2008) Contrast-Dependent and Contrast-Independent Spatial Summation of Primary Visual Cortical Neurons of the Cat. Cerebral Cortex, 18, 331-336. http://dx.doi.org/10.1093/cercor/bhm057

[12] Chen, K., Song, X.-M. and Li, C.-Y. (2013) Contrast-Dependent Variations in the Excitatory Classical Receptive Field and Suppressive Nonclassical Receptive Field of Cat Primary Visual Cortex. Cerebral Cortex, 23, 283-292. http://dx.doi.org/10.1093/cercor/bhs012

[13] Agaian, S.S., Panetta, K. and Grigoryan, A.M. (2000) A New Measure of Image Enhancement. IASTED International Conference on Signal Processing \& Communication, Marbella, September 2000, 19-22.

[14] Fiete, R.D. and Tantalo, T. (2001) Comparison of SNR Image Quality Metrics for Remote Sensing Systems. Optical Engineering, 40, 574-585. 
Scientific Research Publishing (SCIRP) is one of the largest Open Access journal publishers. It is currently publishing more than 200 open access, online, peer-reviewed journals covering a wide range of academic disciplines. SCIRP serves the worldwide academic communities and contributes to the progress and application of science with its publication.

Other selected journals from SCIRP are listed as below. Submit your manuscript to us via either submit@scirp.org or Online Submission Portal.
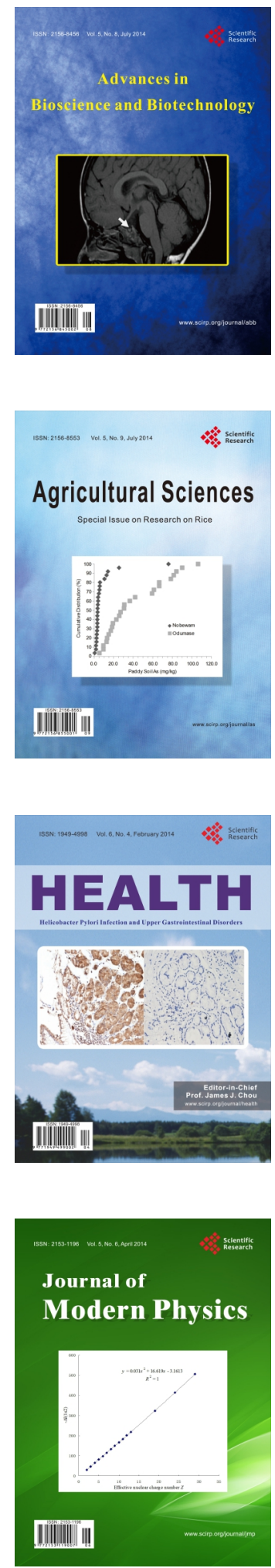
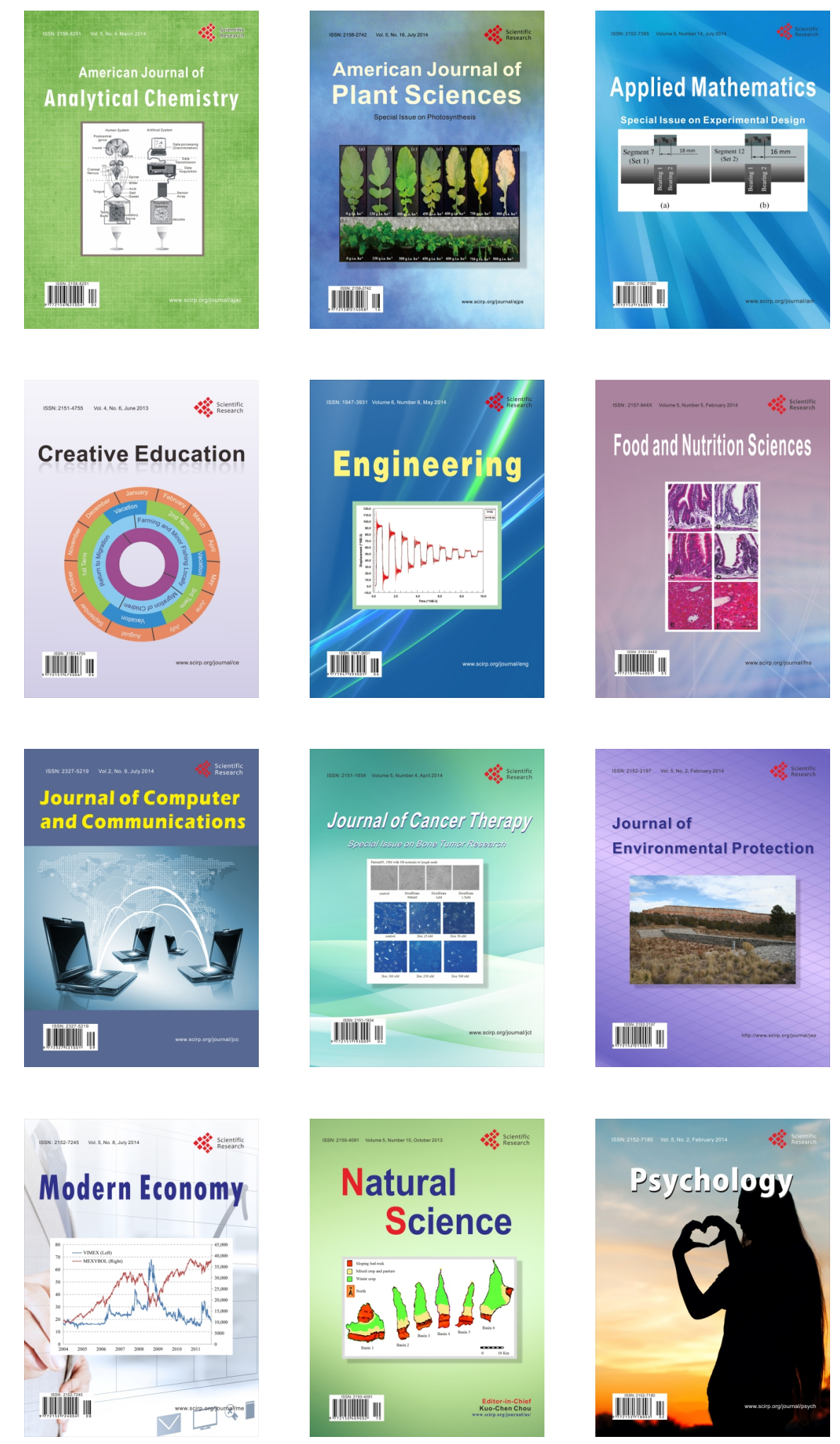